\title{
Irregular semi-convex gradient systems perturbed by noise and application to the stochastic Cahn-Hilliard equation
}

\author{
Giuseppe Da Prato ${ }^{a}$, Arnaud Debussche ${ }^{\mathrm{b}}$, Luciano Tubaro $^{\mathrm{c}}$ \\ a Scuola Normale Superiore di Pisa, Piazza dei Cavalieri 7, 56126, Pisa, Italy \\ b École normale supérieure de Cachan, antenne de Bretagne, campus de Ker Lann, 35170 Bruz, France \\ ${ }^{\mathrm{c}}$ Department of Mathematics, University of Trento, Via Sommarive 14, 38050 Povo, Italy \\ Received 13 January 2003; received in revised form 3 June 2003
}

\begin{abstract}
We prove essential self-adjointness of Kolmogorov operators corresponding to gradient systems with potentials $U$ such that $D U$ is not square integrable with respect the invariant measure (irregular potentials). An application is given to the CahnHilliard-Cook equation in dimension one. In this case the spectral gap is proved for the correspondig semigroup. We also obtain a log-Sobolev inequality.

(c) 2003 Elsevier SAS. All rights reserved.
\end{abstract}

\section{Résumé}

On étudie certains opérateurs de Kolmogorov associés à des systèmes de type gradient ayant un potentiel $U$ tel que $D U$ n'est pas de carré intégrable par rapport à la mesure invariante (potentiels irréguliers). On montre que ceux-ci sont essentiellement auto-adjoints. On applique ensuite les résultats obtenus au cas de l'équation de Cahn-Hilliard-Cook en dimension 1. Dans ce cas, une inégalité de type log-Sobolev est établie ainsi que l'existence d'un trou spectral pour le semigroupe associé. (c) 2003 Elsevier SAS. All rights reserved.

\section{Introduction and setting of the problem}

Let $H$ be a separable real Hilbert space (norm $|\cdot|$, inner product $\langle\cdot, \cdot\rangle$ ). We are concerned with the following Kolmogorov operator

$$
N_{0} \varphi=\frac{1}{2} \operatorname{Tr}\left[D^{2} \varphi(x)\right]+\langle x, A D \varphi(x)\rangle-\langle D U, D \varphi\rangle, \quad \varphi \in \mathcal{E}_{A}(H)
$$

where $D$ denotes the Fréchet derivative with respect to $x$. Here $A: \mathcal{D}(A) \subset H \rightarrow H$ is a negative self-adjoint operator such that $A^{-1}$ is of trace class and $U: H \rightarrow(-\infty,+\infty]$ is a semi-convex function. $\operatorname{Moreover} \mathcal{E}_{A}(H)$ is the vector space of all linear combinations of functions of the form

$$
\cos (\langle x, h\rangle), \sin (\langle x, h\rangle), \quad h \in \mathcal{D}(A) .
$$

E-mail address: tubaro@science.unitn.it (L. Tubaro). 
Let $\mu$ be the Gaussian measure in $H$ with mean 0 and covariance operator $Q:=-\frac{1}{2} A^{-1}$, we consider the measure

$$
v(d x)=Z^{-1} \mathrm{e}^{-2 U(x)} \mu(d x)
$$

where $Z$ is a normalization constant:

$$
Z=\int_{H} \mathrm{e}^{-2 U(x)} \mu(d x),
$$

Our goal is to show that, under suitable assumptions on $U$, the operator $N_{0}$ is dissipative in some space $L^{p}(H, v), p \geqslant 1$ and that its closure is $m$-dissipative.

As well known, the Kolmogorov operator $N_{0}$ is related to a gradient system described by the following differential stochastic equation

$$
d X=(A X-D U(X)) d t+d W(t), \quad X(0)=x,
$$

where $W(t)$ is a cylindrical Wiener process on $H$.

Several papers have been devoted to gradient systems. We recall the Dirichlet forms approach, [1,2,14], and the semigroup approach, see [12] and references therein. But in all these papers, with the exception of [2], ${ }^{1}$ the assumption that (at least) $D U$ is square integrable with respect to $v$ :

$$
\int_{H}|D U(x)|^{2} v(d x)<+\infty
$$

is made. This assumption is fulfilled in several applications as the reaction-diffusion equations, but it does not hold for semilinear equations perturbed by noise where the nonlinearity involves the derivative of the unknown, see [10] for a discussion on this point. In [10] a concrete case, the Kolmogorov equation corresponding to the $p$-laplacian (perturbed by a bilaplacian) was considered. In the present paper we replace (1.3) with the weaker condition

$$
\int_{H}\left|(-A)^{-\frac{1}{2+2 \beta}} D U\right|^{2+2 \beta} d v<+\infty,
$$

where $0 \leqslant \beta \leqslant 1$, proving that the closure $N_{1+\beta}$ of the operator $N_{0}$ is $m$-dissipative in $L^{1+\beta}(H, v)$. As an application, we solve the Kolmogorov equation corresponding to the stochastic Cahn-Hilliard equation.

Let us explain our method. Proceeding as in [9], we consider an approximating equation

$$
\lambda \varphi_{\alpha}-\frac{1}{2} \operatorname{Tr}\left[D^{2} \varphi_{\alpha}(x)\right]-\left\langle x, A D \varphi_{\alpha}(x)\right\rangle+\left\langle D U_{\alpha}, D \varphi_{\alpha}\right\rangle=f
$$

where $f \in \mathcal{E}_{A}(H), \lambda>0$, and $U_{\alpha}$ is a smooth approximation of $U$. We prove that $\varphi_{\alpha} \in D\left(N_{1+\beta}\right)$, so that it can be written as

$$
\lambda \varphi_{\alpha}-N_{1+\beta} \varphi_{\alpha}=f+\left\langle D U-D U_{\alpha}, D \varphi_{\alpha}\right\rangle .
$$

Now the key point is to show that

$$
\lim _{\alpha \rightarrow 0}\left\langle D U-D U_{\alpha}, D \varphi_{\alpha}\right\rangle=0 \quad \text { in } L^{1+\beta}(H, v),
$$

so that the range of $\lambda-N_{1+\beta}$ is dense in $L^{1+\beta}(H, v)$ and $N_{1+\beta}$ is $m$-dissipative. In [9], (1.7) was proved using (1.3) and the basic inequality

$$
\int_{H} N_{0} \varphi \varphi d \nu=-\frac{1}{2} \int_{H}|D \varphi|^{2} d \nu, \quad \varphi \in \mathcal{E}_{A}(H),
$$

\footnotetext{
${ }^{1}$ In [2], $D U$ is not assumed to be square integrable with respect to the $H$-norm but with respect to a weaker norm. However $D^{2} U$ has to be semibounded with respect to the corresponding dual norm, a condition, in general, not easy to check in the applications. We thank the referee for pointing out this fact.
} 
which yields easily an a-priori estimate for $\int_{H}|D \varphi|^{2} d \nu$. In the present situation, since only (1.4) holds, (1.8) is no longer sufficient. We need a stronger estimate which is proved in Section 3.

Section 2 is devoted to some preliminaries, Section 4 to an application to the stochastic Cahn-Hilliard equation in the interval $[0, \pi]$. In this case we prove that $N_{0}$ is essentially self-adjoint in $L^{2}(H, v)$. Moreover, we prove the Poincaré and the $\log$-Sobolev inequalities for the measure $v$. This implies that the spectral gap property holds for $\mathrm{N}_{2}$.

We notice that the Poincaré and the log-Sobolev inequalities do not follow from the Bakry-Emery criterion, see [3], due to the lack of regularity of the potential $U$ of the Cahn-Hilliard equation. The main idea to prove these inequalities is to show that $v$ is the image of a measure $\nu_{0}$ through the embedding $L^{2}([0, \pi]) \subset H^{-1}([0, \pi])$ where $\nu_{0}$ is the invariant measure for a reaction-diffusion system for which the Poincare and the log-Sobolev inequalities hold.

\section{Preliminaries}

Let us state our assumptions. Concerning $A$ we shall assume that

\section{Hypothesis 2.1.}

(i) $A$ is self-adjoint and there exists $\omega>0$ such that

$$
\langle A x, x\rangle \leqslant-\omega|x|^{2}, \quad x \in \mathcal{D}(A) .
$$

(ii) $A^{-1}$ is of trace class.

Remark. From (ii) it follows that there exist a complete orthonormal system $\left\{e_{k}\right\}$ in $H$ and a sequence of positive numbers $\left\{\alpha_{k}\right\}$ such that

$$
A e_{k}=-\alpha_{k} e_{k}, \quad k \in \mathbb{N} \text {, with } \sum_{k \in \mathbb{N}} \frac{1}{\alpha_{k}}<+\infty .
$$

We consider the operator $N_{0}$ as a perturbation of the Ornstein-Uhlenbeck operator $L$

$$
L \varphi(x)=\frac{1}{2} \operatorname{Tr}\left[D^{2} \varphi(x)\right]+\langle x, A D \varphi(x)\rangle, \quad x \in H, \varphi \in \mathcal{E}_{A}(H),
$$

that is

$$
N_{0} \varphi=L \varphi(x)-\langle D U, D \varphi\rangle, \quad \varphi \in \mathcal{E}_{A}(H) .
$$

We recall that $L$ is a self-adjoint operator in $L^{2}(H, \mu)$ with the property that

$$
\int_{H} L \varphi \psi d \mu=-\frac{1}{2} \int_{H}\langle D \varphi, D \psi\rangle d \mu, \quad \varphi, \psi \in W^{1,2}(H, \mu) .
$$

Concerning $U$ we shall make two assumptions.

\section{Hypothesis 2.2.}

(i) Given $U: H \rightarrow(-\infty,+\infty]$, there exists $\delta>0$ such that the function $x \rightarrow U(x)+\delta|x|^{2}$ is convex.

(ii) The number $Z$ defined by (1.2) is finite and positive. 
(iii) There exists a family $\left\{U_{\alpha}\right\}_{\alpha>0}$ of $C^{2}$ class functions such that $x \rightarrow U_{\alpha}(x)+\delta|x|^{2}$ is convex, $U_{\alpha}(x) \leqslant U(x)$ and $U_{\alpha}(x) \uparrow U(x)$ for any $x \in H$.

We shall denote by $v_{\alpha}$ the Borel measure in $H$ defined as

$$
v_{\alpha}(d x)=Z_{\alpha}^{-1} \mathrm{e}^{-2 U_{\alpha}(x)} \mu(d x),
$$

where

$$
Z_{\alpha}:=\int_{H} \mathrm{e}^{-2 U_{\alpha}(x)} \mu(d x) .
$$

\section{Hypothesis 2.3.}

(i) $\lim _{\alpha \rightarrow 0}(-A)^{-\frac{1}{2+2 \beta}} D U_{\alpha}=:(-A)^{-\frac{1}{2+2 \beta}} D U$ in $L^{2+2 \beta}(H, v ; H)$.

(ii) $\lim _{\alpha \rightarrow 0} \int_{H}\left|(-A)^{-\frac{1}{2+2 \beta}} D U_{\alpha}-(-A)^{-\frac{1}{2+2 \beta}} D U\right|^{2+2 \beta} d v_{\alpha}=0$.

(iii) If $\beta=0$, we also assume that there exists $\varepsilon>0$ such that $(-A)^{-\frac{1}{2}} D U \in L^{2+\varepsilon}(H, v ; H)$.

We set

$$
\mathcal{N}_{\alpha} \varphi=L \varphi-\left\langle D U_{\alpha}, D \varphi\right\rangle, \quad \varphi \in \mathcal{E}_{A}(H) .
$$

Lemma 2.4. The following identity holds

$$
\int_{H} \mathcal{N}_{\alpha} \varphi \psi d v_{\alpha}=-\frac{1}{2} \int_{H}\langle D \varphi, D \psi\rangle d v_{\alpha}, \quad \varphi, \psi \in \mathcal{E}_{A}(H) .
$$

In particular, taking $\psi=1$, we get

$$
\int_{H} \mathcal{N}_{\alpha} \varphi d v_{\alpha}=0, \quad \varphi \in \mathcal{E}_{A}(H),
$$

that is $v_{\alpha}$ is infinitesimally invariant for $\mathcal{N}_{\alpha}$.

Proof. Let $\varphi, \psi \in \mathcal{E}_{A}(H)$. We have by (2.1)

$$
\begin{aligned}
\int_{H} L \varphi \psi d v_{\alpha} & =\int_{H} L \varphi\left(\psi \rho_{\alpha}\right) d \mu=-\frac{1}{2} \int_{H}\left\langle D \varphi, D\left(\psi \rho_{\alpha}\right)\right\rangle d \mu \\
& =-\frac{1}{2} \int_{H}\langle D \varphi, D \psi\rangle d v_{\alpha}+\int_{H}\left\langle D \varphi, D U_{\alpha}\right\rangle \psi d v_{\alpha}=0,
\end{aligned}
$$

and the conclusion follows.

We can now prove that the measure $v$ is infinitesimally invariant for $N_{0}$.

Proposition 2.5. We have

$$
\int_{H} N_{0} \varphi d v=0, \quad \varphi \in \mathcal{E}_{A}(H)
$$


and

$$
\int_{H} N_{0} \varphi \varphi d \nu=-\frac{1}{2} \int_{H}|D \varphi|^{2} d \nu, \quad \varphi \in \mathcal{E}_{A}(H) .
$$

Proof. It is enough to prove (2.4), (2.5) follows if we take $\varphi^{2}$ in (2.4). But this follows from (2.3) letting $\alpha$ tend to 0 and taking into account Hypothesis 2.3(ii).

Proposition 2.6. $N_{0}$ is dissipative in $L^{1+\beta}(H, v)$.

Proof. The proof is standard, see [13].

\section{3. m-dissipativity of $N_{1+\beta}$}

Let us first note that, thanks to Proposition 2.6, $N_{0}$ is closable in $L^{1+\beta}(H, v)$; we denote by $N_{1+\beta}$ its closure. We are going to show that $N_{1+\beta}$ is $m$-dissipative.

Let $\alpha, \lambda>0, f \in C_{b}^{1}(H)$ and consider the approximating equation

$$
\lambda \varphi_{\alpha}-L_{1+\beta} \varphi_{\alpha}+\left\langle D U_{\alpha}, D \varphi_{\alpha}\right\rangle=f, \quad \lambda>0 .
$$

Lemma 3.1. If $\lambda>\delta$ Eq. (3.1) has a unique solution $\varphi_{\alpha} \in C_{b}^{1}(H) \cap \mathcal{D}\left(N_{1+\beta}\right)$ and

$$
N_{1+\beta} \varphi_{\alpha}(x)=L_{1+\beta} \varphi_{\alpha}(x)-\left\langle D U(x), D \varphi_{\alpha}(x)\right\rangle, \quad x \in H,
$$

and

$$
\left\|D \varphi_{\alpha}\right\|_{0} \leqslant \frac{1}{\lambda-\delta}\|D f\|_{0}
$$

where $\|\cdot\|_{0}$ denotes the sup norm.

Proof. Step 1. $\varphi_{\alpha} \in C_{b}^{1}(H)$ and (3.3) holds.

It is well known that

$$
\varphi_{\alpha}(x)=\int_{0}^{\infty} \mathrm{e}^{-\lambda t} \mathbb{E}\left[f\left(X_{\alpha}(t, x)\right)\right] d t,
$$

where $X_{\alpha}(t, x)$ is the solution to the following stochastic differential equation

$$
d X_{\alpha}=\left(A X_{\alpha}-D U_{\alpha}\left(X_{\alpha}\right)\right) d t+d W_{t}, \quad X_{\alpha}(0)=x .
$$

Then for any $h \in H$

$$
\left\langle D \varphi_{\alpha}(x), h\right\rangle=\int_{0}^{\infty} \mathrm{e}^{-\lambda t} \mathbb{E}\left[\left\langle D f\left(X_{\alpha}(t, x)\right), \eta_{\alpha}^{h}(t, x)\right\rangle\right] d t,
$$

where $\eta_{\alpha}^{h}(t, x)$ is the solution to the following equation

$$
\frac{d}{d t} \eta_{\alpha}^{h}=A \eta_{\alpha}^{h}-D^{2} U_{\alpha}\left(X_{\alpha}\right) \cdot \eta_{\alpha}^{h}, \quad \eta_{\alpha}^{h}(0)=h .
$$


Consequently $\varphi_{\alpha} \in C_{b}^{1}(H)$. Moreover, multiplying both sides of equation (3.5) by $\eta_{\alpha}^{h}$ and taking in account the dissipativity of $A$ and the convexity of $x \rightarrow U_{\alpha}(x)+\delta|x|^{2}$, yields

$$
\left|\eta_{\alpha}^{h}\right| \leqslant \mathrm{e}^{\delta t}|h|, \quad t>0
$$

Using (3.4) we get

$$
\left|\left\langle D \varphi_{\alpha}(x), h\right\rangle\right| \leqslant \frac{1}{\lambda-\delta}|h|\|D f\|_{0},
$$

and (3.3) is proved.

Step 2. $\varphi_{\alpha} \in \mathcal{D}\left(L_{1+\beta}\right)$ where $L_{1+\beta}$ is the infinitesimal generator of the Ornstein-Uhlenbeck semigroup in $L^{1+\beta}(H, \mu)$,

$$
R_{t} \varphi(x)=\int_{H} \varphi\left(\mathrm{e}^{t A} x+y\right) N\left(0, Q_{t}\right)(d y), \quad \varphi \in C_{b}(H),
$$

where

$$
Q_{t}=-\frac{1}{2} A^{-1}\left(1-\mathrm{e}^{2 t A}\right), \quad t \in[0,+\infty] ;
$$

and observing that $Q_{\infty}=Q$.

We need a further approximating equation:

$$
\lambda \varphi_{\alpha, \beta}-L_{1+\beta} \varphi_{\alpha, \beta}+\frac{1}{1+\beta\left|D U_{\alpha}\right|^{2}}\left\langle D U_{\alpha}, D \varphi_{\alpha, \beta}\right\rangle=f, \quad \lambda>0 .
$$

By [12, Proposition 6.6.4], Eq. (3.6) has a unique solution $\varphi_{\alpha, \beta} \in C_{b}(H)$. Moreover

$\left\|\varphi_{\alpha, \beta}\right\|_{0} \leqslant\|f\|_{0}$,

and there exists $C>0$ such that

$$
\left\|D \varphi_{\alpha, \beta}\right\|_{0} \leqslant \frac{C}{\lambda-\delta}\|f\|_{1} .
$$

Since $D U_{\alpha}$ has linear growth, there exists $C\left(\alpha,\|f\|_{1}\right)>0$ such that

$$
\left|L_{1+\beta} \varphi_{\alpha, \beta}(x)\right| \leqslant C\left(\alpha,\|f\|_{1}\right)(1+|x|), \quad x \in H .
$$

It follows that

$$
\int_{H}\left|L_{1+\beta} \varphi_{\alpha, \beta}(x)\right|^{2} \mu(d x) \leqslant C\left(\alpha,\|f\|_{1}\right)(1+\operatorname{Tr} Q) .
$$

By a standard argument this implies that $\varphi_{\alpha} \in \mathcal{D}\left(L_{1+\beta}\right)$.

Step 3. $\varphi_{\alpha} \in \mathcal{D}\left(N_{1+\beta}\right)$ and (3.2) holds.

Let us first consider the case when $\beta \in(0,1]$. We recall that, see [11],

$$
\left\langle D R_{t} \varphi, h\right\rangle=\int_{H}\left\langle\Lambda(t) h, Q_{t}^{-1 / 2} y\right| \varphi\left(\mathrm{e}^{t A} x+y\right) N\left(0, Q_{t}\right)(d y), \quad \varphi \in L^{p}(H, \mu),
$$

with

$$
\Lambda(t)=Q_{t}^{-1 / 2} \mathrm{e}^{t A} .
$$

Hence,

$$
\left\langle(-A)^{\frac{1}{2+2 \beta}} D R_{t} \varphi, h\right\rangle=\int_{H}\left\langle(-A)^{\frac{1}{2+2 \beta}} \Lambda(t) h, Q_{t}^{-1 / 2} y\right\rangle \varphi\left(\mathrm{e}^{t A} x+y\right) N\left(0, Q_{t}\right)(d y)
$$


and, for $p \geqslant 1$,

$$
\begin{aligned}
\left|\left\langle(-A)^{\frac{1}{2+2 \beta}} D R_{t} \varphi, h\right)\right|^{p} \leqslant & \int_{H}\left|\varphi\left(\mathrm{e}^{t A} x+y\right)\right|^{p} N\left(0, Q_{t}\right)(d y) \\
& \times\left(\int_{H}\left|\left\langle(-A)^{\frac{1}{2+2 \beta}} \Lambda(t) h, Q_{t}^{-1 / 2} y\right\rangle\right|^{q} N\left(0, Q_{t}\right)(d y)\right)^{p / q},
\end{aligned}
$$

where $q$ is the conjugate exponent of $p$. It follows that

$$
\left|(-A)^{\frac{1}{2+2 \beta}} D R_{t} \varphi\right|^{p} \leqslant C t^{-p \frac{2+\beta}{2+2 \beta}} R_{t}(\varphi)^{p}(x), \quad \varphi \in L^{p}(H, \mu),
$$

which, integrating with respect to $\mu$ and taking the Laplace tranform, yields

$$
\left\|(-A)^{\frac{1}{2+2 \beta}} D\left(\lambda-L^{1+\beta}\right) f\right\|_{L^{p}(H, \mu)} \leqslant C(\lambda)\|f\|_{L^{p}(H, \mu)}, \quad \varphi \in L^{p}(H, \mu) .
$$

We are now ready to prove that that $\varphi_{\alpha} \in \mathcal{D}\left(N_{1+\beta}\right)$.

Since $\mathcal{E}_{A}(H)$ is a core for $L_{1+\beta}$, see [5], there exists a sequence $\left\{\varphi_{n}\right\} \subset \mathcal{E}_{A}(H)$ such that,

$$
\lim _{n \rightarrow \infty} \varphi_{n}=\varphi_{\alpha}, \quad \lim _{n \rightarrow \infty} L_{1+\beta} \varphi_{n}=L_{1+\beta} \varphi_{\alpha}, \quad \text { in } L^{1+\beta}(H, \mu) .
$$

We claim that

$$
\lim _{n \rightarrow \infty} N_{1+\beta} \varphi_{n}=L_{1+\beta} \varphi_{\alpha}-\left\langle D U(x), D \varphi_{\alpha}\right\rangle \quad \text { in } L^{1+\beta}(H, v),
$$

which proves that $\varphi_{\alpha} \in \mathcal{D}\left(N_{1+\beta}\right)$.

Since by (3.7) we know that $(-A)^{\frac{1}{2+2 \beta}} D \varphi_{n} \rightarrow(-A)^{\frac{1}{2+2 \beta}} D \varphi$ in $L^{1+\beta}(H, \mu)$, it is enough to show, in view of the Vitali theorem, that

$$
\int_{H}\left|\left\langle D U(x), D \varphi_{n}\right\rangle\right|^{1+\beta+\varepsilon} v(d x)
$$

is bounded, for some $\varepsilon>0$. We have in fact

$$
\begin{aligned}
\int_{H} & \left|\left\langle D U(x), D \varphi_{n}\right\rangle\right|^{1+\beta+\varepsilon} v(d x) \\
& \leqslant \int_{H}\left|(-A)^{-\frac{1}{2+2 \beta}} D U(x)\right|^{1+\beta+\varepsilon}\left|(-A)^{\frac{1}{2+2 \beta}} D \varphi_{n}\right|^{1+\beta+\varepsilon} v(d x) \\
& \leqslant\left(\int_{H}\left|(-A)^{-\frac{1}{2+2 \beta}} D U(x)\right|^{2+2 \beta} v(d x)\right)^{\frac{1+\beta+\varepsilon}{2+2 \beta}}\left(\int_{H}\left|(-A)^{\frac{1}{2+2 \beta}} D \varphi_{n}\right|^{\frac{2(1+\beta+\varepsilon)(1+\beta)}{1+\beta-\varepsilon}} v(d x)\right)^{\frac{1+\beta-\varepsilon}{2+2 \beta}} .
\end{aligned}
$$

Now the claim follows from (3.7).

Let us now consider the case $\beta=0$.

Since $\mathcal{E}_{A}(H)$ is a core for $L_{2}$, there exists a sequence $\left\{\varphi_{n}\right\} \subset \mathcal{E}_{A}(H)$ such that,

$$
\lim _{n \rightarrow \infty} \varphi_{n}=\varphi_{\alpha}, \quad \lim _{n \rightarrow \infty} L_{2} \varphi_{n}=L_{2} \varphi_{\alpha}, \quad \text { in } L^{2}(H, \mu) .
$$

It follows that for all $\psi \in \mathcal{D}\left(L_{2}\right)$ we have, see [12, p. 215], $\left|(-A)^{1 / 2} D \psi\right| \in L^{2}(H, \mu)$ and there exists $c>0$ such that

$$
\int_{H}\left|(-A)^{1 / 2} D \psi\right|^{2} d \mu \leqslant c \int_{H}\left|L_{2} \psi\right|^{2} d \mu, \quad \forall \psi \in \mathcal{D}\left(L_{2}\right) .
$$


Consequently, by (3.8) it follows that

$$
\lim _{n \rightarrow \infty}(-A)^{1 / 2} D \varphi_{n}=(-A)^{1 / 2} D \varphi_{\alpha} \quad \text { in } L^{2}(H, \mu ; H),
$$

and there exists $c>0$ such that

$$
\int_{H}\left|(-A)^{1 / 2} D \varphi_{n}\right|^{2} d \mu \leqslant c
$$

We claim that

$$
\lim _{n \rightarrow \infty} N_{0} \varphi_{n}=L_{2} \varphi_{\alpha}-\left\langle D U(x), D \varphi_{\alpha}\right\rangle \quad \text { in } L^{1}(H, v) .
$$

This will imply that $\varphi_{\alpha} \in \mathcal{D}\left(N_{1}\right)$. It is enough to show that

$$
\int_{H}\left|\left\langle D U(x), D \varphi_{n}(x)\right\rangle\right|^{1+\gamma} d v
$$

is bounded, for some $\gamma>0$. We have in fact

$$
\int_{H}\left|\left\langle D U(x), D \varphi_{n}(x)\right\rangle\right|^{1+\gamma} d \nu \leqslant\left(\int_{H}\left|(-A)^{-\frac{1}{2}} D U(x)\right|^{2+\frac{4 \gamma}{1-\gamma}} d v\right)^{\frac{1-\gamma}{2}}\left(\int_{H}\left|(-A)^{\frac{1}{2}} D \varphi_{n}\right|^{2} d \nu\right)^{\frac{1+\gamma}{2}},
$$

hence, because of Hypothesis (2.3)(iii) in the case $\beta=0$, we can apply the Vitali theorem choosing $\gamma=\frac{\varepsilon}{4+\varepsilon}$.

The following identity for $D \varphi_{\alpha}$ is central in the proof of our main result.

Proposition 3.2. Let $f \in C_{b}^{2}(H)$ and let $\varphi_{\alpha}$ be the solution of (3.1). Then we have

$$
\begin{aligned}
& \lambda \int_{H}\left|D \varphi_{\alpha}\right|^{2} d v_{\alpha}+\frac{1}{2} \int_{H} \operatorname{Tr}\left[\left(D^{2} \varphi_{\alpha}\right)^{2}\right] d v_{\alpha}+\int_{H}\left|(-A)^{1 / 2} D \varphi_{\alpha}\right|^{2} d v_{\alpha}+\int_{H}\left\langle D^{2} U_{\alpha} D \varphi_{\alpha}, D \varphi_{\alpha}\right\rangle d v_{\alpha} \\
& \quad=\int_{H}\left\langle D \varphi_{\alpha}, D f\right\rangle d v_{\alpha}=2 \int_{H} f\left(f-\lambda \varphi_{\alpha}\right) d v_{\alpha} .
\end{aligned}
$$

Proof. Let $f \in C_{b}^{1}(H)$ and let $\varphi_{\alpha}$ be the solution of (3.1). Let us differentiate both sides of (3.1) with respect to $D_{k}, k \in \mathbb{N}$, where $D_{k}$ is the derivative in the direction of $e_{k}$. We obtain

$$
\lambda D_{k} \varphi_{\alpha}-L D_{k} \varphi_{\alpha}+\left\langle D U_{\alpha}, D D_{k} \varphi_{\alpha}\right\rangle+\mu_{k} D_{k} \varphi_{\alpha}+\left\langle D D_{k} U_{\alpha}, D \varphi_{\alpha}\right\rangle=D_{k} f .
$$

Multiplying by $D_{k} \varphi_{\alpha}$, integrating with respect to $v_{\alpha}$ and taking into account (2.2), we find that

$$
\begin{aligned}
& \lambda \int_{H}\left|D_{k} \varphi_{\alpha}\right|^{2} d v_{\alpha}+\frac{1}{2} \int_{H}\left|D D_{k} \varphi_{\alpha}\right|^{2} d v_{\alpha}+\mu_{k} \int_{H}\left|D_{k} \varphi_{\alpha}\right|^{2} d v_{\alpha}+\int_{H}\left\langle D D_{k} U_{\alpha}, D \varphi_{\alpha}\right\rangle D_{k} \varphi_{\alpha} d v_{\alpha} \\
& \quad=\int_{H} D_{k} \varphi_{\alpha} D_{k} f d v_{\alpha} .
\end{aligned}
$$

Summing up on $k$ gives, taking again into account (2.2), the conclusion follows. 
Corollary 3.3. There exists $c_{1}>0$ such that for any $f \in C_{b}^{2}(H)$

$$
\int_{H}\left|(-A)^{1 / 2} D \varphi_{\alpha}\right|^{2} d v_{\alpha} \leqslant c_{1}\|f\|_{0}^{2},
$$

where $\varphi_{\alpha}$ is the solution to (3.1).

Theorem 3.4. The closure $N_{1+\beta}$ of $N_{0}$ in $L^{1+\beta}(H, v)$, is $m$-dissipative in $L^{1+\beta}(H, v)$.

Proof. Let $\lambda>\delta, f \in C_{b}^{2}(H), \alpha>0$, and let $\varphi_{\alpha}$ be the solution to (3.1). Since by Lemma $3.1 \varphi_{\alpha} \in \mathcal{D}\left(N_{1+\beta}\right)$ we can write

$$
\lambda \varphi_{\alpha}-N_{1+\beta} \varphi_{\alpha}=\left\langle(-A)^{-1 / 2}\left(D U-D U_{\alpha}\right),(-A)^{1 / 2} D \varphi_{\alpha}\right\rangle+f .
$$

We claim that

$$
\lim _{\alpha \rightarrow 0}\left\langle D U-D U_{\alpha}, D \varphi_{\alpha}\right\rangle=0 \quad \text { in } L^{1+\beta}(H, v) .
$$

This will conclude the proof by applying the classical result of Lumer and Phillips, [15].

Let us prove (3.11). Since $U_{\alpha}(x) \leqslant U(x)$ and $\lim _{\alpha \rightarrow 0} Z_{\alpha}=Z$, Corollary 3.3 implies

$$
\begin{aligned}
\int_{H}\left|(-A)^{1 / 2} D \varphi_{\alpha}\right|^{2} d v & =Z^{-1} \int_{H}\left|(-A)^{1 / 2} D \varphi_{\alpha}\right|^{2} \mathrm{e}^{-2 U(x)} \mu(d x) \\
& \leqslant Z^{-1} \int_{H}\left|(-A)^{1 / 2} D \varphi_{\alpha}\right|^{2} \mathrm{e}^{-2 U_{\alpha}(x)} \mu(d x) \leqslant \frac{Z_{\alpha}}{Z} c_{1} \leqslant c,
\end{aligned}
$$

where $c$ is a suitable positive constant. Then, by the Hölder inequality we obtain,

$$
\int_{H}\left|\left\langle D U-D U_{\alpha}, D \varphi_{\alpha}\right\rangle\right|^{1+\beta} d v \leqslant\left[\int_{H}\left|(-A)^{-\frac{1}{2+2 \beta}}\left(D U-D U_{\alpha}\right)\right|^{2+2 \beta} d v\right]^{\frac{1}{2}}\left[\int_{H}\left|(-A)^{\frac{1}{2+2 \beta}} D \varphi_{\alpha}\right|^{2+2 \beta} d \nu\right]^{\frac{1}{2}} .
$$

Now we use the well known interpolatory estimate

$$
\left|(-A)^{\frac{1}{2+2 \beta}} x\right|^{2+2 \beta} \leqslant C|x|^{2 \beta}\left|(-A)^{\frac{1}{2}} x\right|^{2}, \quad x \in \mathcal{D}\left((-A)^{\frac{1}{2}}\right),
$$

and find

$$
\begin{aligned}
& \int_{H}\left|\left\langle D U-D U_{\alpha}, D \varphi_{\alpha}\right\rangle\right|^{1+\beta} d v \\
& \leqslant C\left[\int_{H}\left|(-A)^{-\frac{1}{2+2 \beta}}\left(D U-D U_{\alpha}\right)\right|^{2+2 \beta} d v\right]^{\frac{1}{2}}\left[\int_{H}\left|D \varphi_{\alpha}\right|^{2 \beta}\left|(-A)^{\frac{1}{2}} D \varphi_{\alpha}\right|^{2} d v\right]^{\frac{1}{2}} \\
& \leqslant \frac{C}{(\lambda-\delta)^{\beta}}\|D f\|_{0}^{\beta}\|f\|_{0}\left(\int_{H}\left|(-A)^{-\frac{1}{2+2 \beta}}\left(D U-D U_{\alpha}\right)\right|^{2+2 \beta} d v\right)^{1 / 2}
\end{aligned}
$$

thanks to (3.3) and Corollary 3.3. The proof is complete thanks to Hypothesis 2.3(i). 


\section{The stochastic Cahn-Hilliard equation}

\section{1. m-dissipativity}

The Cahn-Hilliard equation is a phenomenological model for various types of nonequilibrium phase transitions as the early stage of spinodal decomposition, a physical phenomenon that arises when we rapidly quench an alloy from the stable region (high temperature) to the unstable region (low temperature). Cook took into account also the thermal fluctuations introducing the stochastic Cahn-Hilliard equation, which in the litterature is known also as the Cahn-Hilliard-Cook equation.

This equation has been intensively studied, see e.g. [4,6,7], and the references cited therein.

We will apply the abstract results of Section 3 to the following stochastic Cahn-Hilliard equation:

$$
\left\{\begin{array}{l}
d X=D_{\xi}^{2}\left(-D_{\xi}^{2} X+f(X)\right) d t+d W(t), \quad \text { in }[0,+\infty) \times[0, \pi] \\
\int_{0}^{\pi} X(\xi) d \xi=0 \\
D_{\xi} X(t, 0)=D_{\xi}^{3} X(t, 0)=D_{\xi} X(t, \pi)=D_{\xi}^{3} X(t, \pi)=0 \\
X(0, \cdot)=x
\end{array}\right.
$$

where $W(t)$ is a cylindrical Wiener process on $\mathcal{H}^{-1}$, and where $f \in C^{2}(\mathbb{R})$ is such that

$$
|f(r)| \leqslant a\left(1+|r|^{2 m-1}\right)
$$

for some $a$ and the function

$$
g(r)=\int_{0}^{r} f(s) d s
$$

is semiconvex. Typically $g$ is a polynomial with positive leading coefficient of even order (greater then or equal to 4). In order to avoid technical complications below, we make the additional assumption that $f$ is monotone, however all our results hold in the more general case of the derivative of a semiconvex function.

The stochastic Cahn-Hilliard equation with periodic boundary conditions can be treated in the same way.

In general $X$ denotes concentration, for instance in the case of a binary alloy $(\mathrm{Cu}, \mathrm{Zn}), X$ can be the concentration of $\mathrm{Cu}$. In the deterministic case the Cahn-Hilliard equation has the property that the total concentration - which corresponds to the spatial average of $X-$ is a conserved quantity. Without loss of generality, we assume that this average is zero. It is natural to require that the noise does not destroy this property. Thus we work in spaces of zero average functions and introduce $\mathcal{H}^{1}(0, \pi)$, the space of functions in $H^{1}(0, \pi)$ whose average is zero, and its dual $\mathcal{H}^{-1}(0, \pi)$.

It is natural to study this problem in the space $H=\mathcal{H}^{-1}(0, \pi)$ because, with this choice, the equation is of gradient type and the corresponding transition semigroup is reversible.

We also consider the Hilbert space $\dot{L}^{2}(0, \pi)$ of all square integrable functions $\varphi$ on $[0, \pi]$ with zero average. Its inner product is denoted by $\langle\cdot, \cdot\rangle$.

Let $\left\{e_{k}\right\}_{k \in \mathbb{N}^{*}}{ }^{2}$ be the orthonormal basis on $\dot{L}^{2}(0, \pi)$ defined by

$$
e_{k}(\xi)=(\pi / 2)^{-1 / 2} \cos (k \xi), \quad k \in \mathbb{N}^{*},
$$

and, for any $x \in L^{2}(0, \pi)$, set

$$
x_{k}=\left\langle x, e_{k}\right\rangle, \quad k \in \mathbb{N}^{*} \text {. }
$$

We shall identify $\dot{L}^{2}(0, \pi)$ with $\ell^{2}\left(\mathbb{N}^{*}\right)$ and then we shall consider $\dot{L}^{2}(0, \pi)$ as a subspace of $\mathbb{R}^{\mathbb{N}^{*}}$.

\footnotetext{
$2 \mathbb{N}^{*}=1,2, \ldots$
} 
Moreover, for any $r \in \mathbb{R}$ we shall denote by $\mathcal{H}^{r}$ the subspace of $\mathbb{R}^{\mathbb{N}^{*}}$ of all sequences $x=\left\{x_{k}\right\}_{k \in \mathbb{N}^{*}}$ such that

$$
|x|_{r}^{2}:=\sum_{k \in \mathbb{N}^{*}}\left(1+|k|^{2}\right)^{r}\left|x_{k}\right|^{2}<+\infty .
$$

$\mathcal{H}^{r}$ is a Hilbert space with the inner product

$$
\langle x, y\rangle_{r}:=\sum_{k \in \mathbb{N}^{*}}|k|^{2 r} x_{k} y_{k}, \quad x, y \in \mathcal{H}^{r} .
$$

The corresponding norm is denoted by $|\cdot|_{r}$. Notice that $\dot{L}^{2}(0, \pi)=\mathcal{H}^{0}, \mathcal{H}^{1}(0, \pi)=\mathcal{H}^{1}, \mathcal{H}^{-1}(0, \pi)=\mathcal{H}^{-1}$ and setting

$$
f_{k}(\xi)=\left(1+|k|^{2}\right)^{1 / 2} e_{k}(\xi), \quad k \in \mathbb{N}^{*},
$$

then $\left\{f_{k}\right\}_{k \in \mathbb{N}^{*}}$ is a complete orthonormal basis on $\mathcal{H}^{-1}$. Clearly, if $r_{1} \geqslant r_{2}$,

$$
|x|_{r_{1}} \leqslant|x|_{r_{2}} .
$$

Moreover, we assume that $W(t)$ is the cylindrical Wiener process on $\mathcal{H}^{-1}$ defined (formally) by

$$
W(t)=\sum_{k \in \mathbb{N}^{*}} f_{k} \beta_{k}
$$

where $\left\{\beta_{k}\right\}_{k \in \mathbb{N}^{*}}$ is a sequence of mutually independent standard Brownian motions.

Let us define the linear (unbounded) operators $A$ and $B$ in $H=\mathcal{H}^{-1}$ by setting

$$
B f_{k}=k^{2} f_{k}, \quad k \in \mathbb{N}^{*},
$$

and

$$
A f_{k}=-k^{4} f_{k}, \quad k \in \mathbb{N}^{*} .
$$

Notice that

$$
\mathcal{D}(B)=\mathcal{H}^{1}, \quad \mathcal{D}(A)=\mathcal{H}^{3},
$$

and that $B=(-A)^{1 / 2}$.

Moreover, let us introduce the potential $U: \mathcal{H}^{-1} \mapsto[0,+\infty]$

$$
U(x)= \begin{cases}\int_{0}^{\pi} g(x(\xi)) d \xi, & \text { if } x \in \mathcal{D}(U), \\ +\infty & \text { otherwise }\end{cases}
$$

where

$$
\mathcal{D}(U)=\left\{y \in \dot{L}^{2}([0, \pi]): g(y) \in L^{1}([0, \pi])\right\},
$$

and $g(r)=\int_{0}^{r} f(s) d s$.

We have

$$
D U(x) \cdot y=\int_{0}^{\pi} g(\xi) d \xi .
$$

We denote by $D_{\mathcal{H}^{r}}$ the gradient in $\mathcal{H}^{r}$, then

$$
D_{\mathcal{H}^{-1}} U=(-A)^{1 / 2} D U=B f(x) .
$$

For $r=-1$, we also set $D=D_{\mathcal{H}^{-1}}$. Thus, Eq. (4.1) can be written as

$$
\left\{\begin{array}{l}
d X=(A X-D U(X)) d t+d W(t) \\
X(0)=x
\end{array}\right.
$$


Now we can consider the Kolmogorov operator

$$
N_{0} \varphi(x)=\frac{1}{2} \operatorname{Tr}\left[D^{2} \varphi(x)\right]+\langle x, A D \varphi(x)\rangle-\langle D U(x), D \varphi(x)\rangle, \quad \varphi \in \mathcal{E}_{A}(H),
$$

which we shall write also as

$$
N_{0} \varphi(x)=\frac{1}{2} \operatorname{Tr}\left[D^{2} \varphi(x)\right]+\langle x, A D \varphi(x)\rangle+\langle f(x), B D \varphi(x)\rangle .
$$

We set $\mu=N_{Q}$ where $Q=-\frac{1}{2} A^{-1}$. We have

Theorem 4.1. Let $N_{0}$ be the Kolmogorov operator defined by (4.6), and let $v$ the probability measure defined by (1.1). Then $N_{0}$ is essentially self-adjoint in $L^{2}\left(\mathcal{H}^{-1}, v\right)$.

Proof. We shall apply Theorem 3.4, verifying the required assumptions for $\beta=1$ and $\delta=0$.

Verification of Hypothesis 2.1. It follows from the identity

$$
\operatorname{Tr} Q=\frac{1}{2} \sum_{k \in \mathbb{N}^{*}} k^{-4}<+\infty
$$

Verification of Hypothesis 2.2(ii). For this it is convenient to write $x(\xi)$ in a suitable form. Given $x \in \mathcal{H}^{-1}$ we start from the obvious identity

$$
x(\xi)=\sum_{k \in \mathbb{N}^{*}}\left\langle x, f_{k}\right\rangle_{-1} f_{k}=\frac{1}{\sqrt{2}}\left\langle Q^{-1 / 2} x, \sum_{k \in \mathbb{N}^{*}} \frac{1}{k^{2}} f_{k}(\xi) f_{k}\right\rangle_{-1}=\rho(\xi) W_{\eta_{\xi}}(x),
$$

where $W$ represents the white noise function, $\eta_{\xi}$ is the element in $\mathcal{H}^{-1}$ defined by

$$
\eta_{\xi}=\frac{1}{\sqrt{2} \rho(\xi)} \sum_{k \in \mathbb{N}^{*}} \frac{1}{k^{2}} f_{k}(\xi) f_{k}
$$

and

$$
\rho^{2}(\xi)=\frac{1}{2} \sum_{k \in \mathbb{N}^{*}} \frac{1+k^{2}}{k^{4}} e_{k}(\xi)^{2}
$$

Now we can prove that

$$
Z=\int_{H} \mathrm{e}^{-2 U(x)} \mu(d x)>0 .
$$

For this it is enough to show that

$$
\int_{H} U(x) \mu(d x)<+\infty \text {. }
$$

We have in fact

$$
\begin{aligned}
\int_{H} U(x) \mu(d x) & =\int_{H} \int_{0}^{\pi} g(x(\xi)) d \xi \mu(d x)=\int_{0}^{\pi} d \xi \int_{H} g\left(\rho(\xi) W_{\eta_{\xi}}(x)\right) \mu(d x) \\
& =(2 \pi)^{-\frac{1}{2}} \int_{0}^{\pi} d \xi \int_{-\infty}^{+\infty} \mathrm{e}^{-\frac{r^{2}}{2}} g(\rho(\xi) r) d r<+\infty,
\end{aligned}
$$


in view of (4.2), and Hypothesis 2.2(ii) is fulfilled.

Verification of Hypothesis 2.2(iii).

Let us define approximations $U_{\alpha}$ of $U$. Let $g_{\alpha}$ be the Moreau-Yosida approximations of $g$

$$
g_{\alpha}(r)=\inf \left\{g(s)+\frac{1}{2 \alpha}(r-s)^{2}: s \in \mathbb{R}\right\} .
$$

We set

$$
U_{\alpha}(x)=\int_{0}^{\pi} g_{\alpha}\left((1+\alpha B)^{-1} x(\xi)\right) d \xi, \quad \alpha>0 .
$$

Then $U_{\alpha}$ is of class $C^{2}$. Moreover, $U_{\alpha} \leqslant U$. In fact, since

$$
(1+\alpha B)^{-1} x(\xi)=\int_{0}^{\pi} k(\xi, \eta) x(\eta) d \eta
$$

with $k(\xi, \eta) \geqslant 0$, we have that $\int_{0}^{\pi} k(\xi, \eta) d \eta=1$ : this allows us to apply Jensen inequality to get

$$
g_{\alpha}\left((1+\alpha B)^{-1} x\right) \leqslant g\left((1+\alpha B)^{-1} x\right) \leqslant(1+\alpha B)^{-1} g(x) .
$$

Hence

$$
U_{\alpha}(x) \leqslant U(x) .
$$

Verification of Hypothesis 2.2(iv). Firstly we observe that

$$
D U_{\alpha}(x) \cdot y=\int_{0}^{\pi} g_{\alpha}^{\prime}\left((1+\alpha B)^{-1} x(\xi)\right)(1+\alpha B)^{-1} y(\xi) d \xi,
$$

so that

$$
D_{\mathcal{H}^{-1}} U_{\alpha}=(-A)^{1 / 2} D_{\mathcal{H}^{0}} U_{\alpha}=B(1+\alpha B)^{-1} g_{\alpha}^{\prime}\left((1+\alpha B)^{-1}\right) .
$$

We have to show that

$$
\lim _{\alpha \rightarrow 0} \int_{\mathcal{H}^{-1}}\left|(-A)^{1 / 4} D_{\mathcal{H}^{-1}}\left(U-U_{\alpha}\right)\right|_{-1}^{4} d v=\lim _{\alpha \rightarrow 0} \int_{\mathcal{H}^{-1}}\left|D_{\mathcal{H}^{0}}\left(U-U_{\alpha}\right)\right|_{0}^{4} d v=0 .
$$

In view of the dominated convergence theorem it is enough to show that $\left|D_{\mathcal{H}^{0}} U_{\alpha}\right|_{0}^{4}$ can be estimated, uniformly in $\alpha$, by a $\nu$-integrable function. We have in fact, using the Jensen inequality

$$
\begin{aligned}
\left|D_{\mathcal{H}^{0}} U_{\alpha}\right|_{0}^{4} & =\left(\int_{0}^{\pi} D_{\mathcal{H}^{0}} U_{\alpha}(x)(\xi)^{2} d \xi\right)^{2}=\left(\int_{0}^{\pi} f_{\alpha}\left((1+\alpha B)^{-1} x\right)(\xi)^{2} d \xi\right)^{2} \\
& \leqslant\left(\int_{0}^{\pi} f\left((1+\alpha B)^{-1} x\right)(\xi)^{2} d \xi\right)^{2} \leqslant\left(\int_{0}^{\pi} f(x)(\xi)^{2} d \xi\right)^{2} .
\end{aligned}
$$

It remains to show that

$$
\int_{\mathcal{H}^{-1}}\left(\int_{0}^{\pi} f(x)(\xi)^{2} d \xi\right)^{2} d v<+\infty
$$


We have in fact, thanks to (4.2), and proceeding as in the proof of (4.9),

$$
\begin{aligned}
\int_{\mathcal{H}^{-1}}\left(\int_{0}^{\pi} f(x(\xi))^{2} d \xi\right)^{2} d v & \leqslant \pi \int_{0}^{\pi} d \xi \int_{\mathcal{H}^{-1}} f(x(\xi))^{4} d v \leqslant a \pi\left(\pi+\int_{0}^{\pi} d \xi \int_{\mathcal{H}^{-1}}(x(\xi))^{8 m-4} d v\right) \\
& =a \pi\left(\pi+(2 \pi)^{-\frac{1}{2}} \frac{(8 m-4) !}{2^{4 m-2}(4 m-2) !} \int_{0}^{\pi} \rho(\xi)^{8 m-4} d \xi\right),
\end{aligned}
$$

which is finite. The proof is complete.

\subsection{Spectral gap}

We consider here the invariant measure $v$ of the Cahn-Hilliard-Cook equation (4.5) in $\mathcal{H}^{-1}$, that is

$$
v(d x)=Z^{-1} \exp (-U(x)) \mu(d x),
$$

where $U$ is defined by (4.4); we suppose that $U$ be a convex potential.

We recall that for a sufficiently smooth function $x$, we have the following relationship between the derivatives in $\mathcal{H}^{0}=\dot{L}^{2}(0, \pi)$ and in $\mathcal{H}^{-1}$ :

$$
D_{\mathcal{H}^{-1}} x=B D_{\mathcal{H}^{0}} x \text {. }
$$

Let $T$ be the natural imbedding of $\mathcal{H}^{0}$ into $\mathcal{H}^{-1}$. It is easily checked that the adjoint $T^{\prime}$ of $T$ is given by $T^{\prime} y=-B^{-1} y$.

Let us consider on $\mathcal{H}^{0}$ the Gaussian measure $\mu_{0}=N\left(0, Q_{0}\right)$ with $Q_{0}=-\frac{1}{2} B^{-1}$ and set

$$
v_{0}(d y)=Z_{0}^{-1} \exp (-U(y)) \mu_{0}(d y)
$$

and

$$
Z_{0}=\int_{H} \exp (-U(y)) \mu_{0}(d y)
$$

It is well known, see e.g. [11] that $v_{0}$ is the unique invariant measure of the following stochastic differential equation

$$
d X=(B X-f(X)) d t+d W_{0} .
$$

We need the following lemma

Lemma 4.2. The image measure of $v_{0}$ through the natural imbedding $T: \mathcal{H}^{0} \rightarrow \mathcal{H}^{-1}$ coincides with $v$.

Proof. We first prove that

$$
\mu\left(\mathcal{H}^{0}\right)=1
$$

We have in fact

$$
\int_{\mathcal{H}^{-1}}|x|_{\mathcal{H}^{0}}^{2} \mu(d x)=\int_{\mathcal{H}^{-1}}|\sqrt{B} x|_{\mathcal{H}^{-1}}^{2} \mu(d x)=\operatorname{Tr}\left(B^{-1}\right)<+\infty .
$$

To prove the lemma it is enough to show that for any Borel bounded function $\varphi: \mathcal{H}^{-1} \rightarrow \mathbb{R}$ we have

$$
\int_{\mathcal{H}^{0}} \varphi(y) v_{0}(d y)=\int_{\mathcal{H}^{-1}} \varphi(x) v(d x) .
$$


To prove (4.12) we consider a sequence $\left\{P_{n}\right\}$ of finite dimensional approximations of the identity in $\mathcal{H}^{0}$ and we set $P_{n}^{\prime}=T P_{n}, n \in \mathbb{N}^{*}$. Then by the change of variables formula in finite dimensional spaces, we get

$$
\int_{P_{n} \mathcal{H}^{0}} \varphi\left(P_{n} y\right) \mathrm{e}^{-2 U\left(P_{n} y\right)} N\left(0, P_{n} Q_{0}\right)(d y)=\int_{P_{n}^{\prime} \mathcal{H}^{-1}} \varphi\left(P_{n}^{\prime} x\right) \mathrm{e}^{-2 U\left(P_{n}^{\prime} x\right)} N\left(0, P_{n}^{\prime} Q\right)(d x) .
$$

Now, letting $n$ tend to infinity and taking into account (4.11), we find (4.12).

Let us prove now the Poincaré inequality for the measure $v$.

Theorem 4.3. For any $\varphi \in C_{b}^{1}\left(\mathcal{H}^{-1}\right)$ we have

$$
\int_{\mathcal{H}^{-1}}|\varphi(x)-\bar{\varphi}(x)|^{2} v(d x) \leqslant \frac{1}{2} \int_{\mathcal{H}^{-1}}\left|D_{\mathcal{H}^{-1}} \varphi(x)\right|_{\mathcal{H}^{-1}}^{2} v(d x)
$$

where

$$
\bar{\varphi}=\int_{\mathcal{H}^{-1}} \varphi(x) v(d x)
$$

Proof. It is well known, see [3, Eq. (4.1)], [8, Proposition 2.3], that the Poincaré inequality holds for the measure $\nu_{0}$. Therefore, taking into account that the principal eigenvalue of $-B$ is 1 and that $U$ is convex, for any $\varphi \in C_{b}^{1}\left(\mathcal{H}^{0}\right)$ we have

$$
\int_{\mathcal{H}^{0}}|\varphi(y)-\overline{\bar{\varphi}}(y)|^{2} v_{0}(d y) \leqslant \frac{1}{2} \int_{\mathcal{H}^{0}}\left|D_{\mathcal{H}^{0}} \varphi(x)\right|_{\mathcal{H}^{0}}^{2} v(d y),
$$

where

$$
\overline{\bar{\varphi}}=\int_{\mathcal{H}^{0}} \varphi(y) v_{0}(d y) .
$$

On the other hand we have, by the change of variables formula, that $\overline{\bar{\varphi}}=\bar{\varphi}$. Consequently

$$
\begin{aligned}
\frac{1}{2} \int_{\mathcal{H}^{-1}}\left|D_{\mathcal{H}^{-1}} \varphi(x)\right|_{\mathcal{H}^{-1}}^{2} v(d x) & =\frac{1}{2} \int_{\mathcal{H}^{-1}}\left|B D_{\mathcal{H}^{0}} \varphi(x)\right|_{\mathcal{H}^{-1}}^{2} v(d x) \\
& \geqslant \frac{1}{2} \int_{\mathcal{H}^{-1}}\left|D_{\mathcal{H}^{0}} \varphi(x)\right|_{\mathcal{H}^{0}}^{2} v(d x)=\frac{1}{2} \int_{\mathcal{H}^{0}}\left|D_{\mathcal{H}^{0}} \varphi(x)\right|_{\mathcal{H}^{0}}^{2} v_{0}(d x) \\
& \geqslant \int_{\mathcal{H}^{0}}|\varphi(x)-\bar{\varphi}(x)|^{2} v_{0}(d x)=\int_{\mathcal{H}^{-1}}|\varphi(x)-\bar{\varphi}(x)|^{2} v(d x),
\end{aligned}
$$

by the change of variables formula.

The spectral gap follows now easily, see [8, Proposition 4.1].

Corollary 4.4. Let $N_{2}$ be the closure of $N_{0}$ in $L^{2}(H, v)$ and let $\sigma\left(N_{2}\right)$ be its spectrum. Then we have $\sigma\left(N_{2}\right) \backslash\{0\} \subset\{\lambda \in \mathbb{C}: \operatorname{Re} \lambda<-1\}$. 
In the same way we obtain the log-Sobolev inequality.

Theorem 4.5. For any $\varphi \in C_{b}^{1}\left(\mathcal{H}^{-1}\right)$ we have

$$
\int_{\mathcal{H}^{-1}} \varphi^{2} \log \varphi^{2} d v \leqslant \int_{\mathcal{H}^{-1}}\left|D_{\mathcal{H}^{-1}} \varphi(x)\right|_{\mathcal{H}^{-1}}^{2} d v+\int_{\mathcal{H}^{-1}} \varphi^{2} d v \log \left(\int_{\mathcal{H}^{-1}} \varphi^{2} d v\right) .
$$

Remark 4.6. As already mentioned, all our results continue to hold if we assume that the nonlinear term $f$ in (4.1) is the derivative of a semiconvex function, which is the case if $f$ is a polynomial of odd degree with positive leading coefficient. In this case, in the proof of Theorem 4.1 we have to choose $\delta>0$. The construction of the approximations $U_{\alpha}$ also has to be modified. The proof of Theorem 4.3 and 4.5 do not use this assumption since it is known that $v_{0}$ satisfy the spectral property and a log-Sobolev inequality also in that case. Of course, in Theorem 4.3, if $U$ is only semiconvex the constant $\frac{1}{2}$ has to be changed to another constant which depends on the oscillations of $U$.

\section{References}

[1] S. Albeverio, R. Høegh-Krohn, Dirichlet forms and diffusion processes on rigged Hilbert spaces, Z. Wahrsch. Geb. 40 (1977) 1-57.

[2] S. Albeverio, V. Kondratiev, M. Röckner, Dirichlet operators via stochastic analysis, J. Funct. Anal. 128 (1) (1995) $102-138$.

[3] D. Bakry, L'hypercontractivité et son utilisation en théorie des semi-groupes, in: Lectures on Probability Theory, Lecture Notes in Math., vol. 1581, Springer, 1994

[4] D. Blömker, S. Majer-Paape, T. Wanner, Spinodal decomposition for the Cahn-Hilliard-Cook equation, Comm. Math. Phys. 223 (2001) $553-582$.

[5] A. Chojnowska-Michalik, B. Goldys, On regularity properties of nonsymmetric Ornstein-Uhlenbeck semigroup in $L^{p}$ spaces, Stoch. Stoch. Reports 59 (1996) 183-209.

[6] C. Cardon-Weber, Cahn-Hilliard stochastic equations: strict positivity of the density, Stochastic and Stochastic Reports 72 (2002) 191277.

[7] G. Da Prato, A. Debussche, Stochastic Cahn-Hilliard equation, Nonlinear Anal. 26 (2) (1996) 241-263.

[8] G. Da Prato, A. Debussche, B. Goldys, Some properties of invariant measures of nonsymmetric dissipative stochastic systems, Probab. Theory Related Fields 123 (3) (2002) 355-380.

[9] G. Da Prato, L. Tubaro, Some results about dissipativity of Kolmogorov operators, Czechoslovak Math. J. 51 (4) (2001) 685-699.

[10] G. Da Prato, L. Tubaro, On a class of gradient systems with irregular potentials, in: Infinite Dimensional Analysis, in: Quantum Probability and Related Topics, vol. 4, 2001, pp. 183-194.

[11] G. Da Prato, J. Zabczyk, Ergodicity for Infinite Dimensional Systems, in: London Mathematical Society Lecture Notes, vol. 229, Cambridge University Press, 1996.

[12] G. Da Prato, J. Zabczyk, Second Order Partial Differential Equations in Hilbert Spaces, in: London Mathematical Society Lecture Notes, vol. 293, Cambridge University Press, 2002.

[13] A. Eberle, Uniqueness and Non-Uniqueness of Singular Diffusion Operators, in: Lecture Notes in Mathematics, vol. 1718, Springer-Verlag, 1999.

[14] V. Liskevich, M. Röckner, Strong uniqueness for a class of infinite dimensional Dirichlet operators and application to stochastic quantization, Ann. Scuola Norm. Sup. Pisa Cl. Sci. 27 (1999) 69-91.

[15] G. Lumer, R.S. Phillips, Dissipative operators in a Banach space, Pacific J. Math. 11 (1961) 679-698. 